\title{
STRAIN RELAXATION INDUCED RED SHIFT OF PHOTOLUMINESCENCE OF CdZnSe/ZnSe QUANTUM WIRES*
}

\author{
H. Straub, G. Brunthaler, W. Faschinger, G. Bauer \\ Institut für Halbleiterphysik, Universität Linz, 4040 Linz, Austria
}

AND C. VIEU

Laboratoire des Microstructures et de Microélectronique CNRS 196 Avenue H. Ravera, 92225 Bagneux, France

Wire patterns $(80-1000 \mathrm{~nm})$ of molecular beam epitaxy grown $\mathrm{Cd}_{0.2} \mathrm{Zn}_{0.8} \mathrm{Se} / \mathrm{ZnSe}$ quantum well were fabricated by a $\mathrm{CH}_{4} / \mathrm{H}_{2}$ reactive ion etching technique. Photoluminescence emission shows with decreasing lateral size a broadening of line shape and a spectral red shift. Calculations for the change of the band gap due to strain relaxation show that this shift of the photoluminescence emission for narrow $\mathrm{Cd}_{0.2} \mathrm{Zn}_{0.8} \mathrm{Se} / \mathrm{ZnSe}$ structures (lattice mismatch of $1.34 \%$ ) can be explained by a partial elastic strain relaxation of the biaxially compressively strained $\mathrm{Cd}_{0.2} \mathrm{Zn}_{0.8}$ Se quantum well after the patterning process.

PACS numbers: 78.55.Et

In nanostructures, formed from heteroepitaxial layers with different bulk lattice constants, the strain plays an important role. It limits the layer thickness in pseudomorphic structures and is also the origin of a tetragonal lattice distortion in the 2D systems for growth along a [001] direction. The biaxial strain leads to energetic shifts of band edges as well as to splittings of the twofold degenerate valence band states of the cubic lattice $[1,2]$.

The influence of strain and strain relaxation on the optical properties of II-VI nanostructures is so far not investigated in detail in the literature. An observed red shift in the photoluminescence (PL) of reactive ion etching (RIE) fabricated $\mathrm{CdZnSe} / \mathrm{ZnSe}[3]$ wires was attributed to strain relaxation but without a detailed investigation.

Here, we present PL investigations and strain calculations for $\mathrm{Cd}_{0.2} \mathrm{Zn}_{0.8} \mathrm{Se} / \mathrm{ZnSe}$ nanostructures. The fabricated wire structures had lateral sizes from $1000 \mathrm{~nm}$ down to $80 \mathrm{~nm}$. We consider the PL emission shäpe and its red shift

\footnotetext{
*This work was supported by the Gesellschaft für Mikroelektronik, Austria.
} 
with decreasing lateral structure size and demonstrate that the origin of this spectral shift is due to strain relaxation.

The investigated sample was grown by molecular beam epitaxy on a GaAs (001) substrate. The $\mathrm{CdZnSe} / \mathrm{ZnSe}$ structure consists of a $1.1 \mu \mathrm{m}$ thick, fully relaxed $\mathrm{ZnSe}$ buffer, a $100 \AA \mathrm{Cd}_{0.2} \mathrm{Zn}_{0.8}$ Se quantum well $(\mathrm{QW})$ and a $100 \mathrm{~nm}$ $\mathrm{ZnSe}$ cap layer. Well defined arrays of $40 \mu \mathrm{m} \times 40 \mu \mathrm{m}$ with wires (oriented along the [110] direction) of different lateral sizes were obtained all on the same sample by electron beam nanolithography and RIE with a $\mathrm{CH}_{4} / \mathrm{H}_{2}$ gas mixture.

Low temperature $(T=1.9 \mathrm{~K}) \mathrm{PL}$ measurements were performed with a mapping setup having a spatial resolution of about $30 \mu \mathrm{m}$ (laser spot diameter). The $\mathrm{Cd}_{0.2} \mathrm{Zn}_{0.8} \mathrm{Se} \mathrm{QW}$ embedded in $\mathrm{ZnSe}$ barriers $\left(E_{\mathrm{g}}=2.82 \mathrm{eV}\right)$ was resonantly excited with an $\mathrm{Ar}^{+}$ion laser at an energy of $2.708 \mathrm{eV}(457.9 \mathrm{~nm})$ with about $100 \mathrm{~W} / \mathrm{cm}^{2}$. The PL spectra of the $\mathrm{Cd}_{0.2} \mathrm{Zn}_{0.8} \mathrm{Se} / \mathrm{ZnSe}$ wires and a $2 \mathrm{D}$ reference mesa are shown in Fig. 1a. With decreasing lateral size the PL intensity decreases
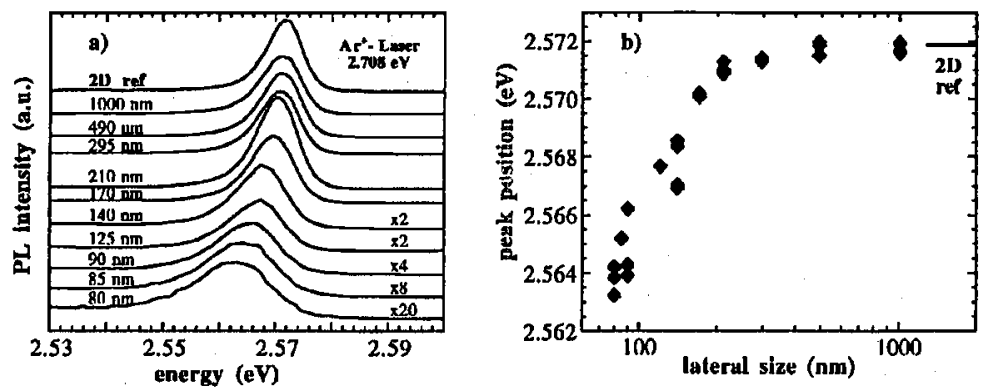

Fig. 1. (a) Photoluminescence spectra (at $1.9 \mathrm{~K}$ ) of $\mathrm{Cd}_{0.2} \mathrm{Zn}_{0.8} \mathrm{Se} / \mathrm{ZnSe}$ quantum wires with sizes from 80 to $1000 \mathrm{~nm}$ and of a 2D reference QW. (b) Red shift of the peak position versus lateral size.

starting at $170 \mathrm{~nm}$ wide wires, the FWHM broadens from $8 \mathrm{meV}$ to about $16 \mathrm{meV}$ and the peaks shift by $8 \mathrm{meV}$ to lower energies. This red shift of the peak positions is shown in more detail in Fig. 1b. For wire sizes below $300 \mathrm{~nm}$ the PL energies become smaller with decreasing lateral size.

The $\mathrm{Cd}_{0.2} \mathrm{Zn}_{0.8}$ Se alloy is fully biaxially (compressively) strained to the lattice constant of the much thicker ZnSe buffer. The size of the symmetric in-plane strain in the layer is given by $\varepsilon_{\|}=\left(a_{\mathrm{ZnSe}}-a_{\mathrm{CdZnSe}}\right) / a_{\mathrm{CdZnSe}}$. With the lattice constants for cubic CdSe $a_{0}=6.052 \AA$ and ZnSe $a_{0}=5.6684 \AA$ [4] interpolated for $\mathrm{Cd}_{0.2} \mathrm{Zn}_{0.8}$ Se one gets an in-plane strain for the $\mathrm{QW}$ layer of $\varepsilon_{\|}=-0.0134$.

Due to the formation of lateral wire structures, one expects additional lateral confinement in the $\mathrm{Cd}_{0.2} \mathrm{Zn}_{0.8}$ Se plane. For $80 \mathrm{~nm}$ wires, this confinement energy is smaller than $1 \mathrm{meV}$ as calculated by a separation ansatz in the effective mass model. This confinement energy (blue shift) is much smaller than the experimentally observed red shifts and will not give a relevant contribution.

As the lateral translation invariance in the QW plane is broken by the partial removal of the material by etching, the structure undergoes an elastic distortion in 
the vicinity of the etched side walls. When the lateral size becomes comparable with the thickness of the strained QW layer, this distortion affects the entire volume of the wire. The geometry of a relaxed wire structure is schematically shown in the inset of Fig. 2b.
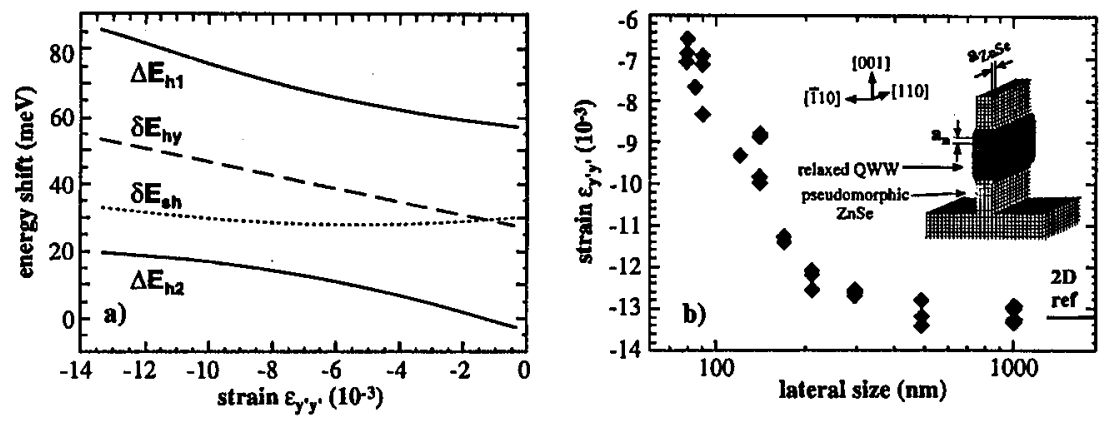

Fig. 2. (a) Energy shift of hydrostatic component $\delta E_{\text {hy }}$ (dashed line), shear component $\delta E_{\mathrm{sh}}$ (dotted line), former light hole $\Delta E_{\mathrm{h} 1}$ (upper solid line) and former heavy hole $\Delta E_{\mathrm{h} 2}$ (lower solid line) for wires with respect to the unstrained situation versus strain $\varepsilon_{y^{\prime} y^{\prime}}$. (b) Averaged strain $\varepsilon_{y^{\prime} y^{\prime}}$ versus lateral size. The inset shows a scheme of a relaxed $\mathrm{ZnSe} / \mathrm{CdZnSe} / \mathrm{ZnSe}$ wire structure.

The $\mathrm{Cd}_{0.2} \mathrm{Zn}_{0.8}$ Se wires are directed along the [110] direction. Therefore the tensor of the elastic constants has to be transformed from the crystallographic reference system into the wire system [5]. We choose the notation $x, y$ and $z$ for [100], [010] and [001] directions, respectively, where the $z$-direction is perpendicular to the layers (i.e. the growth direction). The directions [110] along and $[-110]$ in-plane-perpendicular to the wires are denoted by $x^{\prime}$ and $y^{\prime}$, respectively.

As the wires are very long, a relaxation along the wire ( $x^{\prime}$ direction) is not possible and the relation $\varepsilon_{x^{\prime} x^{\prime}}=\varepsilon_{\|}$holds. The amount of relaxation perpendicular to the wires depends on the lateral width of the wires and is in general not constant across the wire. However, for the purpose of our calculations, we assume a laterally constant effective strain $\varepsilon_{y^{\prime} y^{\prime}}$ which is equivalent to a strain averaged over the lateral dimension $y^{\prime}$. The averaged value of $\varepsilon_{z^{\prime} z^{\prime}}$ can be expressed as

$$
\varepsilon_{z^{\prime} z^{\prime}}=-\frac{c_{12}}{c_{11}}\left(\varepsilon_{x^{\prime} x^{\prime}}+\varepsilon_{y^{\prime} y^{\prime}}\right) \text {. }
$$

In the limit, when the lateral width of the wires is smaller than the thickness of the QW layer, the wire will totally relax in $y^{\prime}$ direction. In that special case the solutions for $\varepsilon_{y^{\prime} y^{\prime}}$ is given by De Caro and Tapfer [6]: $\varepsilon_{y^{\prime} y^{\prime}}=-3 \times 10^{-4}$.

The total change in energy of the band gaps can be described by analytically solving the strain Hamiltonian given by Singh [1]:

$$
\begin{aligned}
& \Delta E_{\mathrm{h} 1,2}=a\left(1-\frac{c_{12}}{c_{11}}\right)\left(\varepsilon_{y^{\prime} y^{\prime}}+\varepsilon_{\|}\right) \\
& \quad \pm \frac{1}{2} \sqrt{b^{2}\left(2 \frac{c_{12}}{c_{11}}+1\right)^{2}\left(\varepsilon_{y^{\prime} y^{\prime}}+\varepsilon_{\|}\right)^{2}+d^{2}\left(\varepsilon_{y^{\prime} y^{\prime}}-\varepsilon_{\|}\right)^{2}},
\end{aligned}
$$


where $\Delta E_{\mathrm{h} 1,2}$ denotes the change of band gaps between conduction band (CB) and light and heavy hole states. In Eq. (2) $a, b$ and $d$ are deformation potentials and $c_{11}$ and $c_{12}$ are elastic constants. The energy eigenstates $\Delta E_{\mathrm{h} 1}$ and $\Delta E_{\mathrm{h} 2}$ are generally mixed states of the light and heavy hole basis set. For a purely tetragonal distortion (the same strain in $x$ and $y$ direction for 2D) without any shear strain, $\Delta E_{\mathrm{h} 1}$ corresponds to the light hole and $\Delta E_{\mathrm{h} 2}$ to the heavy hole states as in this case only a splitting of the levels occurs and no mixing $[1,2]$.

Equation (2) can also be written as a sum of a component $\delta E_{\text {hy }}$ due to hydrostatic pressure and a component $\delta E_{\text {sh }}$ due to tetragonal or lower distortions and shear deformation [2]:

$$
\Delta E_{\mathrm{h} 1,2}=\delta E_{\mathrm{hy}} \pm \delta E_{\text {sh }} \text {. }
$$

For the calculation of band gap changes according to Eq. (2) and (3) one needs the elastic constants and deformation potentials of the materials involved. For $\mathrm{Cd}_{0.2} \mathrm{Zn}_{0.8}$ Se the elastic constants of $\mathrm{ZnSe}$ were extrapolated to the values at $2 \mathrm{~K}\left(c_{11}=94 \mathrm{GPa}, c_{12}=57 \mathrm{GPa}\right.$ and $c_{44}=41 \mathrm{GPa}$ [4]). The values for the deformation potentials are $a=-5.04 \mathrm{eV}$ and $b=-1.12 \mathrm{eV}$ for $\mathrm{Cd}_{0.2} \mathrm{Zn}_{0.8} \mathrm{Se}$ [7]. For $d$ no values are reported, therefore the $\mathrm{ZnSe}$ value of $-3.8 \mathrm{eV}$ [4] was used.

In Fig. 2a, the change of the band gap corresponding to the effective strain $\varepsilon_{y^{\prime} y^{\prime}}$ according to Eq. (2) and (3) is shown. The energies are calculated in the range from fully strained $\left(\varepsilon_{y^{\prime} y^{\prime}}=\varepsilon_{\|}\right)$to fully relaxed structures $\left(\varepsilon_{y^{\prime} y^{\prime}}=-3 \times 10^{-4}\right)$. The energies $\Delta E_{\mathrm{h} 1,2}=\delta E_{\mathrm{hy}} \pm \delta E_{\mathrm{sh}}$ do not depend linearly on the strain component $\varepsilon_{y^{\prime} y^{\prime}}$. The reason is that only the hydrostatic component depends linearly on $\varepsilon_{y^{\prime} y^{\prime}}$, but in the $\delta E_{\text {sh }}$ term the tetragonal distortion and the shear term $\left(\varepsilon_{y^{\prime} y^{\prime}}-\varepsilon_{\|}\right) / 2$ are included and the latter increases from zero for fully strained wires to a maximum for fully relaxed wires. PL occurs for the lowest transition energy which is in this case the transition from CB to the former heavy hole. Starting from $\varepsilon_{y^{\prime} y^{\prime}}=\varepsilon_{\|}$(2D) which is $20 \mathrm{meV}$ higher than for unstrained material with increasing relaxation (smaller $\varepsilon_{y^{\prime} y^{\prime}}$ ) the transition energy decreases until for fully relaxed wires it is $3 \mathrm{meV}$ lower than the unstrained situation.

We now compare the calculated band gap changes with the experimentally observed PL line shifts. The PL from the unetched 2D reference structure corresponds to the band gap of the fully strained $\mathrm{Cd}_{0.2} \mathrm{Zn}_{0.8} \mathrm{Se} \mathrm{QW}$. This energy is by $20 \mathrm{meV}$ higher than that for unstrained bulk material. Due to the formation of narrow wires, the strain in the QW can partly relax and the band gap decreases as calculated. This decrease is observed as a red shift of the PL emission line as shown in Fig. 1. We are now able to assign an effective strain $\varepsilon_{y^{\prime} y^{\prime}}$ to any observed red shift by setting it equal to the calculations represented in Fig. 2a. The result is shown in Fig. 2b. From the 2D reference mesa down to about $500 \mathrm{~nm}$, no significant relaxation of the structures is observed. For smaller structures a steady decrease in the effective strain is observed. For the smallest structures of $80 \mathrm{~nm}$ we get an effective strain of about $6.8 \times 10^{-3}$, this is approximately $50 \%$ of the full $2 \mathrm{D}$ strain of $13.4 \times 10^{-3}$. An extrapolation in Fig. $2 \mathrm{~b}$ indicates that around $25 \mathrm{~nm}$ the effective strain would be zero.

By assigning an effective strain to the $\mathrm{Cd}_{0.2} \mathrm{Zn}_{0.8} \mathrm{Se} / \mathrm{ZnSe}$ wire structures we are able to explain the observed PL red shift quantitatively. In reality the strain is 
not homogeneous over the structure width. Close to the side walls, the relaxation is larger than in the center. As a larger relaxation is connected with a decrease in the band gap, a diffusion of the free carriers from the center to the regions near the side walls will take place. It is therefore difficult to estimate the efficiency of the PL contributions from different positions inside the wire structures. The broadening of the FWHM indicates that this inhomogeneous relaxation takes place and that in the measurement light from all loci in the wires is collected.

In summary, quantum wires of $\mathrm{CdZnSe}$ quantum well structures have been fabricated by electron beam nanolithography and $\mathrm{CH}_{4} / \mathrm{H}_{2}$ reactive ion etching. The quantum wires with lateral width between 80 and $1000 \mathrm{~nm}$ were investigated by $\mathrm{PL}$ at $1.9 \mathrm{~K}$. The observed red shift of the PL emission is explained by a partial relaxation of the biaxial compressive strain in the pseudomorphic quantum well for narrow structures.

\section{References}

[1] J. Singh, in: Handbook on Semiconductors, Ed. T.S. Moss, Vol. 2, Ed. of series M. Balkanski, Elsevier Science, Amsterdam 1994, p. 235ff.

[2] F.H. Pollak, in: Semiconductors and Semimetals, Ed. T.P. Pearsall, Vol. 32, Academic, New York 1990, p. 17.

[3] W. Walecki, W.R. Patterson, A.V. Nurmikko, H. Luo, N. Samarth, J.K. Furdyna, M. Kobayashi, S. Durbin, R.L. Gunshor, Appl. Phys. Lett. 57, 2641 (1990).

[4] Numerical Data and Functional Relationships in Science and Technology, Ed. O. Madelung, Landolt-Börnstein, New Series, Group III, Vol. 17b, Springer Verlag, Berlin 1982 and Vol. 22a, Springer Verlag, Berlin 1987.

[5] E. Anastassakis, E. Liarokapis, Phys. Status Solidi B 149, K1 (1988).

[6] L. De Caro, L. Tapfer, Phys. Rev. B 49, 11127 (1994).

[7] W. Huang, F.C. Jain, Proc. SPIE 2346, 50 (1994). 